\title{
P04-34. Identification of 4E I 0-like antibodies in plasma of an HIV-I CRF02_AG infected patient with broad neutralizing activity using peptide phage libraries
}

\author{
T Dieltjens*, B Willems, L Heyndrickx and G Vanham
}

Address: Microbiology, Institute of Tropical Medicine, Antwerp, Belgium

* Corresponding author

from AIDS Vaccine 2009

Paris, France. 19-22 October 2009

Published: 22 October 2009

Retrovirology 2009, 6(Suppl 3):P62 doi:10.1 186/1742-4690-6-S3-P62

This abstract is available from: http://www.retrovirology.com/content/6/S3/P62

(C) 2009 Dieltjens et al; licensee BioMed Central Ltd.

\section{Background}

Monoclonal antibody 4E10, directed against the MPER region of gp41, is the most broadly neutralizing antibody described to date. Only few HIV-1 infected individuals having 4E10-like Abs in their plasma are identified. Using phage display peptide libraries, mimotopes of the HIV envelope epitopes to which antibodies are raised, can be identified. We investigated an HIV-1 CRF02_AG infected individual with broad cross-neutralizing antibodies $(\mathrm{BCN})$, as determined in a pseudovirus (TZMbl) assay.

\section{Methods}

M13 phage display peptide libraries (New England Biolabs) were used to screen BCN plasma of the HIV-1 positive individual, referred to as ITM4, using a stringent selection strategy. Sequence analysis was performed to identify HIV-1 specific mimotopes. The mimotopes were localized in the ITM4-Env sequence.

\section{Results}

Phage with a NWFxxTxxLxxx motif were selected. This mimotope shares key amino acid residues of the 4E10 epitope, and binds HuMAb 4E10. Antibody reactivity to the 4E10 mimotope was conserved over time (20012007). Pseudovirions were made from 4 different follow up plasma samples from this patient. The pseudovirions from earlier time points are sensitive to the MPER-Abs $4 \mathrm{E} 10$ and 2F5, in the later ones resistance occurs. Also the autologous neutralization results suggest a viral escape from the patient's immune responses. Genetic analysis of the HIV-1 envelope of the follow up samples revealed no remarkable changes in the MPER-regions, suggesting other regions to be responsible for the emerging resistance. The contribution of the 4E10-like Abs to the neutralizing capacity of the patient was evaluated using 4E10sensitive and 4E10 resistant pseudovirions.

\section{Conclusion}

Screening M13 phage display peptide libraries with ITM4 BCN plasma has allowed the identification of antibodies directed to the 4E10 epitope. The NWFxxTxxLxxx mimotope binds $4 \mathrm{E} 10$ antibodies or antibodies sharing the 4E10 epitope. The anti-gp41 Abs seem to contribute to the broad cross-neutralizing capacity of ITM 4. 